\title{
Schwerpunkte einer Gesundheitspolitik für die neue Wahlperiode
}

\section{CHRISTOPHER HERMANN, FRANZ KNIEPS, HARTMUT REINERS}

Dr. Christopher Hermann war bis Ende 2019 Vorstandsvorsitzender der AOK Baden-Württemberg

Franz Knieps ist Vorstand im BKK Dachverband e.V. und Mitherausgeber der Zeitschrift Gesundheits- und Sozialpolitik

Hartmut Reiners ist Ökonom und Publizist und war viele Jahre Referatsleiter im Gesundheitsministerium des Landes Brandenburg

\author{
Der im Februar 1990 veröffentlichte Endbericht \\ der Enquete-Kommission des Bundestages zur \\ Strukturreform der gesetzlichen Krankenversicherung \\ (GKV) war Grundlage für wichtige Reformen im \\ Gesundheitswesen. Einige ihrer Empfehlungen warten \\ immer noch auf die Umsetzung. Dazu gehört vor \\ allem die Integration der Versorgungsstrukturen. \\ Der Vorsitzende der Enquete Kommission war \\ Klaus Kirschner. Er wird am 4. November 2021 \\ 80 Jahre alt. Wir widmen ihm diesen Beitrag.
}

Am 20. Januar 2021 twitterte Bundesgesundheitsminister Jens Spabn: „Unser Gesundheitswesen ist eines der besten der Welt, selbst in einer Pandemie können wir uns darauf verlassen." Sicher, die medizinische Versorgung von Covid-19-Patientinnen und Patienten funktioniert relativ gut, auch wenn manche Intensivstation an ihre Grenzen gestoßen ist. Gemessen an der Zahl der Arztpraxen, der Krankenhausbetten und der medizinischen Geräte ist das deutsche Gesundheitswesen tatsächlich internationale Spitze. Aber das sind nur unzulängliche Qualitätsindikatoren. Die Versorgungsstrukturen sind eine Melange aus Mangel und Überfluss sowie einer schlecht organisierten politischen Verantwortung. Eine integrierte gesundheitliche Versorgung mit einer patientenorientierten Kooperation insbesondere von Arztpraxen und Krankenhäusern und eine verantwortliche politische Zuordnung für die Planung und die Sicherstellung der gesamten Versorgung gibt es im deutschen Gesundheitswesen weiterhin nicht.

In den vergangenen 30 Jahren haben zwei Gesetze das deutsche Gesundheits- wesen strukturell verändert: das Ende 1992 verabschiedete Gesundheitsstrukturgesetz (GSG) und das GKV-Modernisierungsgesetz (GMG) vom November 2003. Das GSG führte insbesondere die freie Kassenwahl für alle in der GKV versicherungsberechtigten Personen ein, flankiert durch einen bundesweiten $\mathrm{Ri}$ sikostrukturausgleich (RSA). Das GMG wies den Weg in ein modernes Leistungsrecht der GKV mit dem Grundsatz der evidenzbasierten Medizin, lockerte in der ambulanten Versorgung das Behandlungsmonopol von niedergelassenen Arztpraxen und flexibilisierte das Vertragsrecht zwischen Krankenkassen und Leistungserbringern. Alle anderen Eingriffe, auch das GKV-Wettbewerbsstärkungsgesetz (GKV-WSG) vom März 2007, müssen eher als Folgegesetze oder Ergänzungen zu den mit GSG und GMG gelegten Pfaden eingeordnet werden.

\section{Eine neue Enquete-Kommission?}

Das GSG und das GMG konnten sich auf den im Februar 1990 veröffentlichten Endbericht der Enquetekommission Strukturreform der gesetzlichen Kranken- 
versicherung stützen, die im Juni 1987 vom Bundestag auf Antrag der SPDFraktion eingerichtet worden war. Unter dem Vorsitz von Klaus Kirschner (SPD) erarbeiteten jeweils neun Abgeordnete der Bundestagsfraktionen und Sachverständige $^{1}$ zusammen eine Bestandsaufnahme der Probleme im Gesundheitswesen, aus denen Reformvorschläge entwickelt wurden. Die gesundheits- und ordnungspolitischen Po-

\section{Die Alternative zu einem} „Big Bang" kann nicht die von Jens Spahn als Bundesgesundheitsminister produzierte Abfolge von weitgehend zusammenhanglosen Detailänderungen sein.

sitionen der Kommissionsmitglieder waren kontrovers. Dennoch herrschte trotz gelegentlich auch lautstark ausgetragener Debatten ein sachliches Arbeitsklima, für das nicht zuletzt Klaus Kirschner als Vorsitzender sorgte.

Uns wird als mit der Enquete-Kommission verbundenen Personen ${ }^{2}$ gelegentlich die Frage gestellt, ob es angesichts der vielen ungelösten Probleme im Gesundheitswesen nicht sinnvoll wäre, erneut eine solches Gremium zu berufen. Wir halten das allenfalls unter gewissen Voraussetzungen für eine zielführende Idee. Als die Enquete-Kommission im Sommer 1987 ihre Arbeit aufnahm, war das bundesrepublikanische Gesundheitswesen sowohl in der Politik als auch in der Wissenschaft weitgehend „Neuland“. Es ging damals vor allem darum, die zentralen Strukturprobleme des GKV-Systems herauszuarbeiten und öffentlich zu machen. Das ist heute angesichts der akademisch etablierten Gesundheitswissenschaften, regelmäßiger Versorgungsreports etwa von Krankenkassen sowie der Gutachten des Gesundheits-Sachverständigenrates in dieser Form nicht mehr erforderlich. Die Strukturprobleme des deutschen Gesundheitswesens sind hinlänglich bekannt. Der Fokus einer „Enquete 2.0“ müsste darauf gerichtet sein, gangbare Wege zu ihrer Bewältigung aufzuzeigen, die bei aller gesellschaftlich notwendigen Kompromissgestaltung eine nachhaltige Lösungsorientierung im Blick behalten. Insoweit hätte sie einen ganz anderen Auf- trag als vor annähernd 35 Jahren. Heute geht es bei einer Schwerpunktsetzung um gesellschafts-und versorgungspolitische und nicht um primär wissenschaftlich zu beantwortende Fragen.

„Das Gesundheitswesen ist kein Gelände für Vereinfacher“, stellt der Ökonomie-Nobelpreisträger Paul Krugman (2018) in einem Nachruf auf den Ende 2017 verstorbenen Gesundheitsökonomen Uwe Reinhardt fest. ${ }^{3}$ Die Vorstellung von einer großen, das Gesundheitswesen dauerhaft grundlegend neu ordnenden Reform scheitert an der komplizierten politischen Realität, in der es zu wirkungsvollen Abwehrkoalitionen kommen kann, die ein Ziel eint: die Verhinderung der sie jeweils betreffenden Reformen (Knieps und Reiners 2015: 7 ff.). Die Alternative zu einem „Big Bang“ kann aber nicht die von Jens Spahn als Bundesgesundheitsminister produzierte permanente Abfolge von weitgehend zusammenhanglosen Detailänderungen und „Omnibus“-Gesetzen sein (Hermann 2020). Es müssen steuerungspolitische Prioritäten gesetzt werden, die einer systematischen Logik folgen.

\section{1. ÖGD und Public Health}

Die Corona-Pandemie hat den in der $\mathrm{Zu}$ ständigkeit der Länder und Kommunen liegende Öffentliche Gesundheitsdienst (ÖGD) in den Blickpunkt der Öffentlichkeit gerückt. Er war schon lange ein Stiefkind der Gesundheitspolitik. Das Gesetz zur Vereinheitlichung des Gesundheitswesens von 1934 war von der Rassenideologie NS-Deutschlands geprägt und hat den Begriff der „Volksgesundheit“ diskreditiert. Heute spricht man von „Public Health“. Dennoch galt dieses Nazi-Gesetz auch lange Zeit in der Bundesrepublik und wurde erst in den 1980er Jahren durch Ländergesetze ersetzt. Der moderne ÖGD besitzt ein breites Aufgabenspektrum. Es umfasst weiterhin eher Maßnahmen klassischer Eingriffsverwaltungen wie die Bekämpfung von Infektionskrankheiten, aber ebenso gesundheits- und zivilgesellschaftlich-emanzipatorische Aufgabenstellungen wie den schulärztlichen und den sozialpsychiatrischen Dienst, Suchtberatung und Gesundheitsberichterstattung.

Das sind personalintensive Aufgaben, aber es mangelt an der entsprechenden
Ausstattung (Kuhn und Willner 2020). Den 375 deutschen Gesundheitsämtern stehen nach Angaben des Verbandes der Ärzte im ÖGD 17.000 Stellen zur Verfügung, die nicht alle besetzt werden können. Der Bedarf an Ärztinnen und -Ärzten ist im ÖGD weit höher als die gegenwärtige Zahl von gut 2.500 Stellen. Deren Besoldung ist im Vergleich zu Fachärztinnen und -ärzten in Krankenhäusern um 30 bis 50 Prozent niedriger, ganz zu schweigen von den mit einer Arztpraxis potenziell verbundenen Verdienstmöglichkeiten. In der Folge bilden ÖGD-Ärztinnen und Ärzte die einzige Facharztgruppe mit einer rückläufigen Anzahl von Mitgliedern.

Die Gesundheitsämter sind nicht nur personell ausgedünnt, sie sind auch materiell schlecht ausgestattet. Ihre IT-Ausrüstung befand sich während der ersten Wellen der Corona-Pandemie in vielen Kommunen noch im Fax-Zeitalter. Das Deutsche Elektronische Melde-und Informationssystem (DEMIS), das die Kommunikation innerhalb des ÖGD auf den Stand der Technik bringen soll, ist seit 2013 in Arbeit und erst seit kurzem funktionstüchtig. Es gibt auch keine durchgängig brauchbare Software für das in der Pandemiebekämpfung wesentliche Nachverfolgen von Kontakten („,contact tracing“). Diese Lücken in der Ausstattung des ÖGD lassen sich nur schrittweise schließen.

Die Große Koalition hat vor dem Hintergrund der Corona-Pandemie vier Milliarden Euro für die zeitgemäße Ausstattung der Gesundheitsämter bereitgestellt. Allerdings konzentrieren sich diese Mittel auf die Infektionsbekämpfung und lassen andere Aufgaben des ÖGD außen vor. Bei dessen Neugestaltung sollte es auch um den Public-Health-Gedanken gehen, der auf eine enge Verbindung der Prävention mit der kurativen Versorgung abzielt (Holst 2020). Darüber wird zwar in Gre-

1 Zu den Abgeordneten gehörten neben dem Vorsitzenden Klaus Kirschner (SPD) u. a. die Abgeordneten Karl Becker, Paul Hofacker, Editha Limbach (CDU), Horst Seehofer (CSU), Jürgen Egert, Horst Jaunich (SPD), Dieter Thomae (FDP) und Heike WilmsKegel (Die Grünen). Sachverständige waren Prof. Fritz Beske, Prof. Herbert Genzel, Prof. Wolfgang Gitter, Eugen Glombig, Ellis Huber, Prof. Günter Neubauer, Prof. Peter Oberender, Hartmut Reiners und Prof. Rolf Rosenbrock.

2 Christopher Hermann und Franz Knieps waren als wissenschaftliche Mitarbeiter des Sekretariats der Enquete-Kommission an der Erstellung des Berichtes beteiligt, Hartmut Reiners als Mitglied (Sachverständiger).

3 „Health care is no country for simplistic men." 
mien wie der Nationalen Präventionskonferenz der Sozialversicherungsträger oder der Gesundheitsministerkonferenz der Länder (GMK) diskutiert. Aber eine systematische Kooperation des ÖGD mit den Trägern der kurativen Versorgung gibt es nach wie vor nicht. Zudem haben wir erhebliche Zweifel daran, dass die Schaffung von "Gesundheitsregionen“ unter wesentlicher Steuerung kommunaler Einrichtungen, wie sie im grünen Politikmilieu favorisiert wird, an dieser Situation etwas ändern könnte. (Hermann 2021). Der ÖGD hat weder absehbar die Kompetenzen, geschweige denn die Erfahrung in der komplexen Steuerung der medizinischen Versorgung im GKV-System. Schon unabhängig von allen verfassungsund sozialrechtlichen wie ordnungspolitischen Einwendungen würde man den ÖGD maßlos überfordern.

\section{Reform der Versorgungsstrukturen}

Die Segmentierung der medizinischen Versorgung in ambulante und stationäre Einrichtungen und das Fehlen systematischer Strukturen in den Versorgungsketten sind zentrale Mängel unseres Gesundheitswesens. Der Krankenhausreport des Wissenschaftlichen Instituts der AOK (WIdO), der OECD-Report über Verschwendung im Gesundheitswesen und diverse Bestandsaufnahmen (u. a. Bertelsmann Stiftung 2019, SVR-G 2009 und 2018) dokumentieren vor allem folgende Probleme des deutschen Gesundheitswesens:

- Überversorgung mit Krankenhausbetten. Ihre Zahl liegt gemessen an der Bevölkerung um 70 Prozent über dem Durchschnitt vergleichbarer EU-Länder.

- Über fünf Millionen der jährlichen stationären Behandlungsfälle könnten ambulant oder teilstationär versorgt werden.

- Zahlreiche Eingriffe werden von Kliniken durchgeführt, die dafür zu niedrige Fallzahlen ausweisen und nicht über die erforderliche Erfahrung und Ausstattung verfügen.

- Insbesondere in der Fachmedizin rechnen sich Einzelpraxen immer weniger. Die Betriebskosten einer Arztpraxis beanspruchten vor 30 Jahren durchschnittlich 40 Prozent der Einnahmen, heute sind es 60 Prozent (Kostenstrukturanalyse des Statistischen Bundesamtes; Fachserie 2, Reihe 1.6.1).

\section{Flexibilisierung des Vertragsrechts und Kassenwettbewerb}

Die durch das GMG ab 2004 ermöglichten Selektivverträge, die die Krankenkassen ohne Einbindung der Kassenärztlichen Vereinigungen (KV) mit Leistungserbringern schließen können, haben die segmentierten Versorgungsstrukturen nicht wesentlich verändert. Mit der ambulanten spezialärztlichen Versorgung durch Kassenärzte und Krankenhäuser entstanden neue Vertragsbeziehungen, die aber auf definierte schwere Erkrankungen etwa in der Onkologie beschränkt sind und das Monopol der KVen in der ambulanten Versorgung kaum angetastet haben. Die Trennung zwischen ambulanter und stationärer Versorgung konnte nicht überwunden werden. Diese Möglichkeiten gehen zudem mit einer wachsenden Intransparenz einher. Für die ambulante Behandlung in Krankenhäusern existieren zehn verschiedene gesetzliche Grundlagen bzw. Vertragsformen (Leber Wasem 2016).

\section{Im Kassenwettbewerb haben Veränderungen im Vertragsrecht keine Bedeutung, weil sie von allen Krankenkassen einheitlich und gemeinsam bedient werden müssen.}

Im Kassenwettbewerb um Mitglieder haben diese Veränderungen im Vertragsrecht schon deshalb keine Bedeutung, weil sie von allen Krankenkassen einheitlich und gemeinsam bedient werden müssen. Zudem stellte der Gesundheits-Sachverständigenrat in einem Sondergutachten zu den Aufgaben und Zielen des Kassenwettbewerbs schon vor Jahren fest, dass nicht Fragen der Versorgungsqualität den Kassenwettbewerb dominieren, sondern die Höhe der Beitragssätze (SVR-G 2012: Ziffer 488). Diese sei für die Versicherten eine sehr viel transparentere Größe als die schon für Experten schwer messbare Versorgungsqualität. Daraus entsteht ein Dilemma des Kassenwettbewerbs. Der Aufbau integrierter Versorgungsformen ist für Krankenkassen mit Investitionen verbunden, die ihre Früchte allenfalls mittel- bis langfristig tragen. Kurzfristig können sie Druck auf den Zusatzbeitragssatz ausüben, was eine Abwanderung zu
Wettbewerberkassen begünstigt. Hinzu kommt, dass die meisten Versorgungsverträge auf regionaler Ebene geschlossen werden, während insbesondere Ersatzkassen bundesweit agieren und an regionalen Besonderheiten wenig Interesse zeigen. Auch an diesen Strukturen scheitert ein versorgungsorientierter Wettbewerb.

\section{Sicherstellungsauftrag}

Die Vorstellung, der Kassenwettbewerb könne aus sich heraus effektive Anreize für den Aufbau von integrierten Versorgungsformen und Vertragsnetzen bieten, ging stets an der Realität eines in tradierten Organisationsmodellen verharrenden Gesundheitswesens vorbei. Die herrschende Gesundheitspolitik hat dabei um ein zentrales ordnungspolitisches Problem einen Bogen gemacht. Man kann nicht Selektivverträge zwischen Krankenkassen und Leistungserbringern ermöglichen, ohne damit zugleich den Sicherstellungsauftrag der KVen als einen Eckpfeiler des gegenwärtigen GKV-Systems tendenziell auszuhebeln. Dieser ist versorgungsseitig auch mit der morbiditätsbedingten Gesamtvergütung (MGV) verbunden. In dem Maße, wie die MGV durch Selektivverträge der einzelnen Kassen bzw. ihrer Verbände ersetzt wird, geht der Sicherstellungsauftrag (gesetzlich) an die Krankenkassen über. Sie sind allein für ihre eigenen Versicherten verantwortlich, nicht aber für alle Versicherten ganzer Regionen. Ohne weiterführende Regelungen kann ein Vakuum in der Verantwortung für eine flächendeckende Sicherstellung der Versorgung entstehen.

Das Grundgesetz überantwortet den Ländern in Artikel 30 die politische Verantwortung für die allgemeine Daseinsvorsorge, zu der auch die Gewährleistung einer umfassenden gesundheitlichen Versorgung gehört. Daraus wird die $\mathrm{Zu}-$ ständigkeit für die stationäre Versorgung abgeleitet. In der ambulanten Versorgung haben die Länder keine unmittelbaren Durchgriffsrechte, sondern im Wesentlichen allein eine Rechtsaufsicht über KVen und Regionalkassen. Eine organisierte politische Verantwortung für die gesamte medizinische Versorgung existiert nicht.

Soll dies zukünftig verändert werden, könnten die Beziehungen zwischen den politischen Instanzen und den Selbstver- 
waltungsorganen auf Landesebene in einer sektorenübergreifenden Versorgungsplanung neu geordnet werden. Der Gesundheits-Sachverständigenrat hat hierzu in seinem Jahresgutachten 2018 Empfehlungen erarbeitet, die eine Grundlage für die politische Diskussion bilden können (SVR-G 2018: Ziffer 848 ff., Wahlster et al. 2018). Als „konzeptioneller Nukleus“ könnten die Landesgremien nach $\$ 90 \mathrm{a}$ SGB V dienen, die entsprechend umstrukturiert werden müssten. Denkbar wäre eine paritätische Besetzung wie im Gemeinsamen Bundesausschuss, hälftig aus Leistungserbringern (Kassenärztliche Vereinigung und Krankenhäuser) und den Leistungsträgern (GKV und Länder) Die abschließende Entscheidung über die in regelmäßigen Abständen zu erstellenden Versorgungspläne müsste aus verfassungsrechtlichen Gründen bei den Landesparlamenten liegen. Allerdings sollte sich niemand Illusionen machen: Neuordnungen von politischen Zuständigkeiten und deren Institutionen wecken ein ebenso wirkungsmächtiges wie teilweise irrationales Widerstandspotenzial - nicht nur in der Gesundheitspolitik.

\section{Neuordnung von Aufsichtszuständigkeiten}

Eine gemeinsame Verantwortung der Selbstverwaltungsorgane und der Landesbehörden für die Sicherstellung der gesamten medizinischen Versorgung könnte nur dann zielführend sein, wenn Landesbehörden die dafür erforderlichen Kompetenzen in der Aufsicht erhielten. Der Kassenwettbewerb hat die Zentralisierung des GKV-Systems befruchtet. Auch dadurch haben sich Aufsichtskompetenzen zunehmend von der Landesauf die Bundesebene verlagert. Versorgungsverträge werden aber regelmäßig auf Landesebene geschlossen. Das Bundesamt für soziale Sicherung (BAS) erhält so die Aufsicht für regional geschlossene Verträge, ohne dass die Landesbehörden verantwortlich eingebunden werden müssen. Die GMK hat dieses Problem schon vor über zehn Jahren angesprochen (GMK 2010), ohne dem jedoch konkrete Initiativen folgen zu lassen.

Die Zuständigkeitsverteilung zwischen den Aufsichten des Bundes und der Länder steht seit langem in der Debatte. Bereits 2003 hat der damalige Präsident des früheren Bundesversicherungsamtes Rainer Daubenbüchel in einer Bund-
Länder-Arbeitsgruppe vorgeschlagen, die Aufsicht über die Haushalte der Krankenkassen dem Bund zu übertragen, während sich die Länder um das weitgehend auf der regionalen Ebene abspielende Geschäft der Versorgungsverträge kümmern sollten. Dieser Denkanstoß wurde damals u. a. mit dem Hinweis verworfen, eine solche Neuordnung sei politisch kaum durchsetzbar. Inhaltliche Gegenargumente blieben eher rar. Eine solche Reform der Aufsicht erforderte zumindest eine ständige, auch operativ enge Abstimmung zwischen dem BAS und den Aufsichten der Länder, die erheblich über die gegenwärtig vorgegebenen gesetzlichen Abstimmungsprozesse ( $\mathbb{S} 90$ Abs. 4 und 5 SGB IV) hinausgehen müsste.

\section{Finanzierung der GKV}

Schon vor Ausbruch der Covid 19-Pandemie zeichnete sich ab, dass die GKV in ein massives Defizit rutschen wird. 2019 hatte sie erstmals seit zehn Jahren wieder einen Fehlbetrag, der mit 1,9 Mrd. Euro aber angesichts von Rücklagen in Höhe von fast $20 \mathrm{Mrd}$. Euro verkraftbar schien. Diese Reserven sind mittlerweile abgeschmolzen. Die Krankenkassen schätzen das für 2021 zu erwartende Defizit auf 16 Milliarden Euro und für 2022 auf 17 Milliarden Euro. Das Pflegepersonalstärkungsgesetz $(\mathrm{PpSG})$ und das Termin-Service-Gesetz (TSVG) führen danach zu Mehrausgaben von geschätzt 8,2 Milliarden Euro im Jahr. Hinzu kommen Mehrausgaben von jährlich 16 Milliarden Euro, die das Ende 2020 verabschiedete Gesundheitsversorgungsund Pflegeverbesserungsgesetz (GVPG) mit sich bringt. Dafür wurde bereits für das Jahr 2021 der Bundeszuschuss zum Gesundheitsfonds um fünf Milliarden Euro angehoben, weitere acht Milliarden Euro mussten die Krankenkassen aus ihren Rücklagen an den Gesundheitsfonds abführen. Außerdem wurde der durchschnittliche Zusatzbeitragssatz von 1,1 auf 1,3 Prozentpunkte angehoben. Wie es mit der GKV-Finanzierung weitergehen soll, bleibt bis nach den Bundestagswahlen am 29. September 2021 und der Bildung einer neuen Regierung ungewiss. Klar ist indessen, dass die Reserven der GKV erschöpft sind und Beitragssatzstabilität kurz- und mittelfristig nicht einzuhalten ist, wenn die Finanzierungsgrundlagen der GKV unverändert bleiben. Das ergibt sich auch aus den ökonomischen Besonderheiten des Gesundheitswesens als einem personalintensiven Wirtschaftszweig.

\section{Der „Baumol“-Effekt}

Die „Sozialgarantie“ der letzten MerkelRegierung, das Problem einstweilen über erhöhte Zuschüsse des Bundes an den Gesundheitsfonds zu lösen, kann allenfalls eine Übergangsregelung sein. Hier wird ein eigentlich altbekanntes Phänomen übersehen (Reiners 2020). Die Ursache für langfristig steigende GKV-Ausgaben ist auch abseits von medizinisch-technischem Fortschritt oder der demografischen Entwicklung in Deutschland keine fahrlässig verursachte „Kostenexplosion“, sondern sie ist nicht zuletzt Ausdruck ökonomischer Gesetzmäßigkeit. Die Gesundheitswirtschaft unterliegt grundsätzlich einem stärkeren Wachstum als die Volkswirtschaft insgesamt. Ärztliche und pflegerische Leistungen stellen personenbezogene Dienste dar, die wegen ihrer gegenüber der Industrieproduktion geringeren $\mathrm{Ra}-$ tionalisierbarkeit einen immer größeren Anteil des Arbeitskräftepotenzials und der Wertschöpfung moderner Volkswirtschaften beanspruchen.

Der US-Ökonom William Baumol (2012) hat diesen nach ihm benannten Effekt anhand eines Vergleichs der Preisentwicklungen in der Computerindustrie und im Gesundheitswesen dargestellt. Man kann aber auch andere Konsumgüterbereiche als Vergleichsmaßstab wählen. Durchschnittsverdiener mussten in den 1970er Jahren für einen neuen Farbfernseher einen kompletten Monatslohn ausgeben. Heute bekommen sie ein technisch weit besseres Flachbildschirmgerät schon für einen halben Wochenlohn. Ärztliche Behandlungen und pflegerische Dienste kosten heute hingegen deutlich mehr als früher. Baumol bezeichnet diesen Effekt als „Kostenkrankheit“. Aber er ist keine Krankheit, also ein abnormer Zustand, sondern eines der wenigen Axiome der Ökonomie, also ein unter sonst gleichen Bedingungen nicht veränderbarer Sachverhalt.

Der britische Ökonom Jonathan Aldred (2020) zeigt in seinem Buch „Der korrumpierte Mensch“ (S. 143 f.), dass bei einer produktivitätsorientierten Verteilungspolitik die Baumolsche „Kostenkrankheit“ gar nicht so unerfreulich ist, wie sie klingen mag. Durch die Produktivitätszuwächse der Gesamtwirtschaft könnten die Durchschnittslöhne schneller steigen als die Prei- 
se, sodass insgesamt die meisten Güter und Dienstleistungen durch die gestiegene Kaufkraft erschwinglicher würden. Selbst wenn die zweifelhafte Prognose von Baumol stimme, dass die US-Gesundheitsausgaben bis zum Jahr 2100 auf sechzig Prozent des Bruttoinlandsprodukts (BIP)steigen werden, bleibe immer noch genug übrig für einen hohen allgemeinen Lebensstandard, da die meisten Konsumgüter gemessen am BIP billiger werden können.

Grundsätzlich gibt es im deutschen Sozialversicherungssystem unter finanzwirtschaftlichen Aspekten drei Möglichkeiten, mit dem Baumol-Effekt umzugehen: steigende Steuerfinanzierung der GKV-Ausgaben, Anhebung der privaten Finanzierung (Zusatzbeiträge, Zuzahlungen, private $\mathrm{Zu}$ satzversicherungen etc.) oder Erweiterung der finanziellen Basis der GKV.

\section{Holzweg Steuerfinanzierung}

Der Präsident des Bundessozialgerichts (BSG) Rainer Schlegel machte im November 2020 in mehreren Interviews den Vorschlag, die gesetzliche Kranken- und Pflegeversicherung generell von der Beitragsauf eine Steuerfinanzierung umzustellen. Dies wäre ein Nullsummenspiel, in dem sinkende Sozialabgaben mit höheren Steuerbelastungen erkauft werden. Um welche Dimensionen es sich dabei handeln würde, kann ansatzweise bereits einer Antwort der Bundesregierung auf eine Anfrage der Bundestagsfraktion von Bündnis 90 / Die Grünen aus dem Februar 2010 entnommen werden (BT-Drs. 17/258). Der damalige Gesundheitsminister Rösler (FDP) wollte die GKV-Beiträge auf eine einheitliche Kopfpauschale mit einem aus Steuern finanzierten Sozialausgleich für niedrigere Einkommensgruppen umstellen. Die dadurch dem Bundeshaushalt entstehenden Mehrausgaben wurden je nach Höhe der Kopfpauschale damals auf zwischen 22 und 35 Milliarden Euro geschätzt. Diese Beträge dürften heute, zehn Jahre später, deutlich höher liegen.

Würde man dem Vorschlag des BSGPräsidenten folgen und nicht nur einen Sozialausgleich, sondern die gesamten Ausgaben der Kranken- und Pflegeversicherung in Höhe von jährlich 300 Milliarden Euro auf den Bundeshaushalt übertragen, würden sich die erforderlichen Steueranhebungen gegenüber dieser Berechnung um ein Mehrfaches erhöhen. Es ist nicht zu erwarten, dass sich die Belastungen aus Steuern und Sozialabgaben insgesamt verringern wür- den. Sie würden vielmehr lediglich von einem Träger des Sozialbudgets auf einen anderen verlagert, wobei eine Steuerfinanzierung nur dann gegenüber dem Status quo sozial gerechter wäre, wenn sie sich primär aus Einkommen-, Erbschaft- und Vermögenssteuern speisen würde. Die Finanzierung über Umsatz- und Verbrauchssteuern würde untere Einkommensgruppen ungleich härter treffen als einkommensund vermögensstärkere Schichten (Bach et al. 2017).

Die Steuerfinanzierung der Gesundheitsausgaben würde an der gesamten Abgabenbelastung der Wirtschaft und der Bürgerinnen und Bürger per Saldo wenig ändern. Sie ist auch in Deutschland im europäischen Vergleich entgegen anders lautenden Behauptungen nicht auffällig hoch. Der jährliche Survey der OECD (2021) zu den Belastungen der Wirtschaft durch Steuern zeigt jedenfalls, dass sich Deutschland in dem Ranking zur Abgabenbelastung im Mittelfeld bewegt. Die Bundesrepublik weist zwar relativ hohe Sozialabgaben auf, aber diese werden durch geringere Steuern als etwa in den skandinavischen Ländern kompensiert.

\section{Unsinnige „Sozialbremse"}

Der im Sozialgesetzbuch verankerte Grundsatz der Beitragssatzstabilität (\$ 71 SGB V) darf als ein Restposten der gescheiterten „einnahmeorientierten Ausgabenpolitik“ gelten. Er hat nur noch symbolische Bedeutung, auch weil er wegen des BaumolEffekts langfristig nicht zielführend sein kann. Bundeswirtschaftsminister Altmaier und Arbeitgeberverbände übersehen entscheidende ökonomische Zusammenhänge, wenn sie annehmen, dass die Lohnnebenkosten mit einer "Sozialbremse“ von 40 Prozent der sozialversicherungspflichtigen Löhne konstant halten zu können. Der Wortbestandteil „neben“ suggeriert, dass es sich bei den entsprechenden Kosten um im Grunde verzichtbare Ausgaben handelt und die Arbeitskosten ohne die Sozialabgaben entsprechend geringer wären. Das aber ist ein Trugschluss. Er ignoriert den schon vor Jahrzehnten von Piero Sraffa (1976: 28) analysierten „Doppelcharakter der Löhne“, wonach diese einerseits den Lebensunterhalt der Beschäftigten decken müssen und in das Wirtschaftssystem „eingehen wie der Kraftstoff für Maschinen oder das Futter für das Vieh“, andererseits Teile der Überschussproduktion in sich einschließen können.

Bei Tarifverhandlungen geht es demnach sowohl um das Verteilen von Produktivitätszuwächsen als auch um die Kompensation der Lebenshaltungskosten der Arbeitnehmer, zu denen die Sozialabgaben unstreitig gehören. Würden die Sozialabgaben der Arbeitgeber den Versicherten zugeschlagen oder privatisiert, 
nem Jahresgutachten 2004/2005 fest, „dass sowohl in allokativer als auch in distributiver Hinsicht eine Pflichtversicherungsgrenze und die damit verbundene Segmentierung des Krankenversicherungsmarktes ökonomisch nicht begründbar ist“. (SVR-W 2004: Ziffer 494)

Der Schlüssel für eine sozial gerechtere GKV-Finanzierung könnte die Öffnung der GKV für Beamte sein. Sie haben Anspruch auf staatliche Beihilfe zur gesundheitlichen Versorgung, die 50 Prozent der Behandlungskosten erstattet (Pensionäre 70, Kinder 80 bis 100 Prozent). Die Restkosten deckt eine private Krankenversicherung ab. Die Einschätzung, das Beihilfesystem basiere auf der grundgesetzlichen Alimentationsverpflichtung des Staates für seine Beamten (Art. 33 Abs. 6 GG) und sei daher alternativlos, übersieht schon, dass der Staat dieser Verpflichtung auch mit der Zahlung des Arbeitgeberanteils zur GKV nachkommen könnte. Die Abschaffung des Beihilferechts wäre verfassungsrechtlich zulässig (Kingreen/Kühling 2013).

Gegenwärtig kann die GKV lediglich zu einer Option für die Beamten ausgestaltet werden (Kingreen 2018). Hamburg hat seinen Beamten die Möglichkeit eröffnet, zwischen der Beihilfe und einem Zuschuss der Senatsbehörden zum GKV-Beitrag zu wählen, der dem Arbeitgeberanteil entspricht. Andere Länder sind dem Vorgehen gefolgt oder haben die Absicht, diese Regelung zu übernehmen. Da Beamte nicht zu den in der GKV versicherungsberechtigten Personen zählen, kommen dafür gegenwärtig nur Berufsanfänger und Beamte in Frage, die trotz vollständig eigener Beitragsbelastung in der GKV geblieben sind, auch weil sie wegen Vorerkrankungen keinen PKV-Versicherer finden konnten. Eine Studie der Bertelsmann-Stiftung (2016) hat ergeben, dass sich rein rechnerisch die Mitgliedschaft in der GKV für die große Mehrheit der Beamten lohnen würde. Das gilt vor allem für Personen mit Kindern und nicht erwerbstätigen Lebenspartnern.

\section{Ausblick}

Bereits angesichts des interessenverminten Geländes in der Gesundheitspoli- tik sollten Reformschwerpunkte in der neuen Wahlperiode miteinander verbunden in einem durchaus ehrgeizigen, aber abgestuften Prozess umgesetzt werden. Wir plädieren dafür, schwerpunktmäßig endlich die lange überfällige Integration der Versorgungsebenen strukturell zu verankern und mit diesem Ziel die $\mathrm{Zu}$ sammenarbeit von Selbstverwaltung und staatlichen Behörden vor allem auf Länderebene neu zu gestalten. Dabei scheint ein Vorgehen zukunftsfähig zu sein, das die getrennte politische Verantwortung für die ambulante und stationäre Versorgung beendet und in eine gemeinsame Zuständigkeit der Länder und der unmittelbaren Akteure überführt. Das würde auch eine neue Arbeitsteilung zwischen dem Bund und den Ländern in Aufsichtsangelegenheiten bedingen. Ein zweiter Schwerpunkt sollte die generelle Öffnung der GKV für Beamtinnen und Beamte sein als erster Schritt hin zu einem modernen, solidarischen Gesundheitssystem für alle Menschen in der Bundesrepublik.

\section{Literatur}

Aldred, Jonathan 2020: Der korrumpierte Mensch. Die ethischen Folgen wirtschaftlichen Denkens. Stuttgart: Klett-Cotta

Bach, Stefan, Martin Beznoska und Viktor Steiner 2017: Wer trägt die Steuerlast? Verteilungswirkungen des deutschen Steuer- und Transfersystems. Düsseldorf: Hans Böckler Stiftung, Study Nr. 347

Baumol, William (2012): The Cost Disease. Why Computers Get Cheaper and Health Care Doesn't. New Haven, CT: Yale University Press

Bertelsmann Stiftung 2016: Krankenversicherungspflicht für Beamte und Selbständige. Teilbericht Beamte. Bielefeld: Bertelsmann Stiftung.

Bertelsmann Stiftung (Hrsg.) 2019:

Zukunftsfähige Krankenhausversorgung. Gütersloh: Bertelsmann https://www. bertelsmann-stiftung.de/de/publikationen/ publikation/did/zukunftsfaehige-

krankenhausversorgung/

Enquete-Kommission „Strukturreform der gesetzlichen Krankenversicherung “ (1990): Endbericht. Bundestagsdrucksache 11/6380 vom 12.02.1990

Hermann, Christopher 2020: Narrativ im Kaffeesatz opder das GKV-System auf der Rutschbahn des exekutiven Dirigismus. Observer Gesundheit vom 18.09.2020

Hermann, Christopher 2021: Schöne neue grüne Versorgungswelt durch "Gesundheitsregionen"? Oberserver Gesundheit vom 17.06.2021
Holst, Jens 2020: Covid 19: Besondere Herausforderungen an Public Health. Das Gesundheitswesen 82. Jg., Heft 11: 829-835

Kingreen, Thorsten. (2018): Einwohnerversicherung „light“? Reformen und Reformoptionen für die Weiterentwicklung der dualen Krankenversicherungsordnung. Die Krankenversicherung Nr. 2/2018: 45-52

Thorsten Kingreen und Jürgen Kühling 2013: Monistische Einheitsversicherung. BadenBaden-Nomos

Knieps, Franz und Hartmut Reiners 2015: Gesundheitsreformen in Deutschland. Geschichte - Intentionen - Kontroversen. Bern: Huber

Krugman, Paul 2018: A Tribute to Uwe Reinhardt. New York Times, 22 April 2018. https://www.nytimes.com/2018/04/22/ opinion/a-tribute-to-uwe-reinhardt.html

\section{Kuhn, Joseph und Manfred Willner} 2020: Corona-Krise und öffentlicher Gesundheitsdienst. G+G-Wissenschaft 20. Jg., Heft 4: 15-22

Leber, Wulf-Dietrich und Jürgen Wasem 2016: Ambulante Krankenhausleistungen ein Überblick, eine Trendanalyse und einige ordnungspolitische Anmerkungen.

OECD 2017: Tackling Wasteful Spending on Health. https://www.oecd.org/health/ tackling-wasteful-spending-on-health9789264266414-en.htm

OECD 2020: Health at a Glance. https:// ec.europa.eu/health/state/glance_de
OECD 2021: Taxing Wages https://www.oecd. org/tax/taxing-wages-20725124.htm

Reiners, Hartmut 2009: Ideenschmiede für die Gesundheitspolitik. Gesundheit und Gesellschaft 12. Jg., Heft 12: 35-39

Reiners, Hartmut 2020: "Sozialgarantie“ als Perspektive? Anmerkungen zum Vorschlag, die GKV aus Steuern zu finanzieren. Gesundheitsund Sozialpolitik 74. Jg., Heft 6: 49-51

Sraffa, Piero 1976: Warenproduktion mittels Waren. Einleitung zu einer Kritik der politischen Ökonomie. Frankfurt am Main: Suhrkamp

SVR-G (Sachverständigenrat zur Begutachtung des Gesundheitswesens) 2009: Koordination und Integration-Gesundheitsversorgung in einer Gesellschaft des längeren Lebens. Sondergutachten 2009. Bundestags-Drucksache 16/3770 vom 02.07.2009

SVR-G 2012: Wettbewerb an der Schnittstelle zwischen ambulanter und stationärer Versorgung. Sondergutachten 2012. BundestagsDrucksache 17/10232 vom 10.07.2012

SVR-G 2018: Bedarfsgerechte Steuerung der Gesundheitsversorgung. Jahresgutachten 2018. Bundestags-Drucksache 19/3180 vom 04.07.2018

Wahlster, Philip, Sandra Teichmann und David Herr 2018: Wege zur sektorenübergreifenden Versorgungsplanung. G+G-Wissenschaft 18. Jg., Heft 4: 7-15

WIdO (Jürgen Klauber et al.): Krankenhausre port, verschiedene Jahrgänge. https://www. wido.de/publikationen-produkte/buchreihen/ krankenhaus-report/ 